\title{
Patterning of the chick forebrain anlage by the prechordal plate
}

\author{
Edgar M. Pera and Michael Kessel* \\ Max-Planck-Institut für biophysikalische Chemie, D-37077 Göttingen, Germany \\ *Author for correspondence (e-mail: mkessel1@gwdg.de)
}

\section{SUMMARY}

We analysed the role of the prechordal plate in forebrain development of chick embryos in vivo. After transplantation to uncommitted ectoderm a prechordal plate induces an ectopic, dorsoventrally patterned, forebrain-like vesicle. Grafting laterally under the anterior neural plate causes ventralization of the lateral side of the forebrain, as indicated by a second expression domain of the homeobox gene $N K X 2.1$. Such a lateral ventralization cannot be induced by the secreted factor Sonic Hedgehog alone, as this is only able to distort the ventral forebrain medially. Removal of the prechordal plate does not reduce the rostrocaudal extent of the anterior neural tube, but leads to significant narrowing and cyclopia. Excision of the head process results in the caudal expansion of the $N K X 2.1$ expression in the ventral part of the anterior neural tube, while $P A X 6$ expression in the dorsal part remains unchanged. We suggest that there are three essential steps in early forebrain patterning, which culminate in the ventralization of the forebrain. First, anterior neuralization occurs at the primitive streak stage, when BMP-4-antagonizing factors emanate from the node and spread in a planar fashion to induce anterior neural ectoderm. Second, the anterior translocation of organizer-derived cells shifts the source of neuralizing factors anteriorly, where the relative concentration of BMP-4-antagonists is thus elevated, and the medial part of the prospective forebrain becomes competent to respond to ventralizing factors. Third, the forebrain anlage is ventralized by signals including Sonic Hedgehog, thereby creating a new identity, the prospective hypothalamus, which splits the eye anlage into two lateral domains.

Key words: prechordal plate, forebrain, Sonic Hedgehog, chick

\section{INTRODUCTION}

The prechordal plate is a mesendodermal structure underlying the anlage of the forebrain (Adelmann, 1922). Its location in a central position of the head, where endo-, ecto-, neuroecto- and mesoderm are in close proximity, suggests a strategic role in induction and patterning of anterior structures. During gastrulation, the prechordal plate is generated as a direct derivative of the organizer after prospective foregut endoderm has been formed and before the notochord is laid down. In chicken, it is derived from Hensen's node, initially as a relatively small group of mesenchymal cells dorsal to, but also integrated into, the anterior endodermal epithelium. During early forebrain development, the mesenchymal cell population, i.e. the prechordal mesoderm, lies dorsal to the preoral foregut, establishing a close association with both gut and forebrain, anterior to the notochord (Adelmann, 1922; Meier, 1981; Seifert et al., 1993; Sulik et al., 1994). For these stages we define the prechordal mesoderm plus the associated endoderm as the prechordal plate. We exclude from this definition the more anteriorly located endodermal plate, directly adjacent to the ectoderm without invening mesoderm. Finally, the descendants of the prechordal mesoderm are found in certain ocular muscles, as determined in chick-quail chimeras (Couly et al., 1992; Wachtler et al., 1984).

Neural induction is a major event occurring during early head development. Since the discovery of the organizer (Spemann and Mangold, 1924), evidence has accumulated in favour of both planar and vertical induction mechanisms, the former originating from the organizer itself and the latter from its direct derivatives, the prechordal plate, the head process and the notochord (Slack and Tannahill, 1992; Ruiz i Altaba, 1993). Several genes are known to be specifically expressed in the organizer and derived axial mesoderm, including genes encoding homeodomain proteins and secreted factors (De Robertis, 1995). Expressed in the right cells at the right time for neural induction in Xenopus laevis are the secreted proteins follistatin, noggin and chordin, three factors capable of inducing anterior neuroectoderm from naive ectoderm (Hemmati-Brivanlou et al., 1994; Lamb et al., 1993; Sasai et al., 1995; Hemmati-Brivanlou and Melton, 1997). They function by binding directly and thus inactivating TGF $\beta$ related factors, i.e. activin in the case of follistatin, BMP-4 in the case of noggin and chordin (Hemmati-Brivanlou et al., 1994; Piccolo et al., 1996; Yamashita et al., 1995; Zimmermann et al., 1996). Since the same neuralizing factors are produced by the organizer and the axial mesoderm, the molecular mechanisms for planar and vertical induction could be identical. The biological significance of a twofold neural induction event, first planar by the organizer, then vertical by the prechordal plate, is still unclear.

Once a neural plate is induced, it becomes dorso-ventrally 
patterned, as indicated by the rising of the neural folds, the formation of the median hinge point and, later, by the formation of alar and basal plate, as well as roof- and floorplate (Schoenwolf and Smith, 1990). Ventralizing activity is exhibited by the notochord, which induces a floorplate in the posterior forebrain, midbrain, hindbrain and spinal cord (Placzek et al., 1990; Smith and Schoenwolf, 1989; van Straaten et al., 1988; Yamada et al., 1991). This ventralization effect is mediated by the secreted protein signal Sonic Hedgehog (SHH; Echelard et al., 1993; Roelink et al., 1994). Transplanted notochords are not, however, capable of inducing a floorplate in the anterior forebrain anlage (Placzek et al., 1993). On the other hand, an involvement of $\mathrm{SHH}$ in forebrain patterning as well is suggested by several experiments. Targeted disruption of the murine Shh gene impairs formation of the ventral forebrain and leads to a single, fused optic vesicle (Chiang et al., 1996). In rostral neural plate explants $\mathrm{SHH}$ induces the differentiation of ventral forebrain neurons in vitro (Ericson et al., 1995). Overexpression of SHH by injection of RNA into zebrafish embryos results in suppression of the pax6, and ectopic expression of the pax2, axial, $n k 2.2$ and zp50 genes in dorsal parts of the forebrain (Barth and Wilson, 1995; Hauptmann and Gerster, 1996; Krauss et al., 1993; Macdonald et al., 1995).

We have studied the inductive activity of the prechordal plate by transplantation and ablation experiments in cultured chick embryos, which allowed us to suggest an interpretation of the in vivo situation. Use of molecular markers indicated that there are two separate effects on forebrain development. With the formation of the prechordal plate the source of neuralizing factors is translocated rostrally. The neural plate then becomes competent to receive a ventralizing signal, such as $\mathrm{SHH}$.

\section{MATERIALS AND METHODS}

\section{Embryos}

Fertile White Leghorn eggs were obtained from Lohmann Tierzucht, Cuxhaven, Germany, and incubated at $38^{\circ} \mathrm{C}$. Embryos were staged according to Hamburger and Hamilton $(\mathrm{HH}$; Hamburger and Hamilton, 1951). Scanning electron microscopy of the embryos was performed as described (Hayat, 1978).

\section{SHH-expressing cells}

Primary chick embryo fibroblasts (CEFs) from standard specific pathogen-free eggs were cultured and transfected with $S H H$ RCASA2 retroviral DNA (Riddle et al., 1993) as described (Fekete and Cepko, 1993). The cells were harvested 10 days later, after infection had spread through the culture. Anti-gag immunohistochemistry with AMV-3C2 (Potts et al., 1987) (Developmental Studies Hybridoma Bank) and goat anti-mouse Cy3 (Jackson Immuno Research) as primary and secondary antibodies, respectively, confirmed complete infection. CEFs were grown on a tissue culture dish to $50-90 \%$ confluency, scraped, cut into pieces of the same size or up to fivefold that of the prechordal plate graft, and washed in Dulbecco's modified Eagle's medium (DMEM, Gibco). Some of the CEF grafts were labeled with fixative-stable DiI (1,1'-dioctadecyl 3,3,3',3'-tetramethyl indocarbocyanine perchlorate; CellTracker CM-DiI; Molecular Probes, Oregon). Briefly, 0.5\% (w/v) DiI/ethanol was diluted 1:10 in $0.3 \mathrm{M}$ sucrose, loaded onto the graft for 5 minutes at $37^{\circ} \mathrm{C}$ and then for an additional 15 minutes at $4^{\circ} \mathrm{C}$. After loading, the graft was washed in phosphate-buffered saline and DMEM.

\section{Neural plate explant culture}

Rostral neural plate explants corresponding to the presumptive pros- encephalic region were dissected from HH6 chick embryos and cultured either alone or in contact with CEF aggregates for 24-48 hours as described (Ericson et al., 1995).

\section{Transplantations}

Grafts of axial mesendoderm or mesoderm with adhering endoderm were excized from donor embryos (HH5 -6 ), submerged in PannettCompton saline (PCS) with $0.2 \%$ (w/v) Dispase (Boehringer Mannheim), using a sharpened tungsten wire or a bent insect needle. The extent of the grafts was assessed by morphological criteria and expression of SHH, as visualized by in situ analysis (see below). Adjacent paraxial mesenchyme and paraxial endoderm were excluded from the grafted tissue as much as possible. The ectodermal epithelium of the donor remained unaffected, as checked carefully under the dissecting microscope, ensuring that the graft was completely devoid of ectodermal cells. The excized tissue was washed subsequently in 5\% (v/v) fetal calf serum (Gibco)/PCS and DMEM. The grafts were inserted into small pockets between hypoblast and epiblast in the area opaca, or between endoderm and ectoderm in the area pellucida, of host embryos (HH3-8), so that the mesodermal component of the implant faced the ventral side of the host ectoderm. Younger host embryos (up to HH6) were manipulated in a modified New culture, while older ones (HH8) were treated in ovo (Stern, 1993). In the explantation experiment, pieces of axial tissue were removed without including the ectoderm in a modified New culture (Stern, 1993).

\section{Probes and in situ analysis}

Antisense riboprobes labeled with digoxigenin (Boehringer Mannheim) were synthesized from the following chicken cDNAs as templates: NKX2.1 isolated from a HH9 head library (details to be published elsewere), CNOT1 (Stein and Kessel, 1995), EN2 (Gardner et al., 1988), PAX6 (Li et al., 1994), OTX2 (Bally-Cuif et al., 1995) and $\mathrm{SHH}$ (Riddle et al., 1993). Whole-mount in situ hybridization and histology were essentially done as described (Stein and Kessel, 1995; Wilkinson, 1992). Photographs of whole-mount preparations were taken using reflected light from two fiber optic sources. DiI-labeled specimens were documented as double exposures with epifluorescence and bright field optics using Adobe Photoshop (Adobe Systems, Inc.). Sections were photographed using bright field optics.

\section{RESULTS}

\section{Anterior neural induction by the prechordal plate}

We analyzed the inducing potential of the anterior axial mesendoderm or mesoderm on uncommitted ectoderm by transplantation into the rostral inner margin of the area opaca $\left(\mathrm{HH}^{+} / 4^{-}\right)$. This host region is competent to respond to neurulation-inducing signals as shown by grafting experiments with different parts of the primitive streak including the organizer, Hensen's node (Dias and Schoenwolf, 1990; Izpisúa-Belmonte et al., 1993; Lemaire et al., 1997; Storey et al., 1992; Streit et al., 1997).

In order to define the transplanted cell groups precisely, we relied on morphological criteria and the expression of the homeobox genes goosecoid (GSC), CNOT1 and CNOT2 (Izpisúa-Belmonte et al., 1993; Stein and Kessel, 1995; Stein et al., 1996). The first cells of the axial mesoderm, which have ingressed at $\mathrm{HH}^{-}$, are referred to as 'young head process' (YHP). They form a small triangular cell assembly directly anterior to the node and express predominantly $G S C$ and in addition $C N O T 1 / 2$. Due to the anterior migration of $G S C$ expressing cells (Niehrs et al., 1993), these two cell populations segregate quickly. At HH5 the prechordal plate (PP) becomes apparent as a button-shaped structure, only express- 
ing GSC. It is followed by the rod-like head process (HP), with caudally decreasing GSC and increasing CNOT1/2 expression. From HH6 onwards, the notochord is generated, consisting of CNOT1/2-expressing cells only.

The transplantation experiments were analyzed with molecular markers for the chick brain primordium. Nkx2.1 expression is characteristic of the ventral forebrain in mammals (Ericson et al., 1995; Lazzaro et al., 1991; Price et al., 1992; Shimamura et al., 1995). We isolated a chicken homolog (NKX2.1) and detected initial expression at HH8 in the ventral forebrain anlage before closure of the anterior neural folds and appearance of the primary optic vesicles (details to be published elsewhere). In addition, we employed the following well-established molecular markers: CNOT1 as a dorsal forebrain marker (Stein and Kessel, 1995), EN2 as a marker for the midbrain-hindbrain boundary (Gardner et al., 1988) and $P A X 6$ as a marker of the eye primordium in the lateral forebrain ( $\mathrm{Li}$ et al., 1994).

Upon transplantation of young head processes $(n=15)$, slightly elongated vesicular structures formed (Fig. 1A-C, arrowheads). The vesicles arose by a typical neural folding event from the epiblast and consisted of a thick, apparently neural epithelium after around 16 hours of incubation. Expression of NKX2.1, CNOT1 and EN2 indicated that they were of fore- and midbrain identity. Transplantation of prechordal plates (HH5; $n=20$ ) induced neural vesicles, which were positive for NKX2.1 and CNOT1 in distinct expression domains, reflecting dorsoventrally patterned forebrain structures (Fig. 1D,E, arrowheads). No midbrain structure, however, was induced, as demonstrated by the absence

Fig. 1. Induction of anterior neural vesicles by transplantation of anterior axial mesendoderm. Grafts were placed into the area opaca of host embryos $\left(\mathrm{HH}^{+} / 4^{-}\right)$, according to the operation scheme shown in dorsal view at the bottom right. The embryos were analysed at HH10-11 by whole-mount in situ hybridization with riboprobes for NKX2.1 (A,D,G), CNOT1 (B,E) or EN2 (C,F). Dorsal views of the induced structures (arrowheads and arrows) and the anterior part of the primary embryos are shown. Cross sections of whole-mount preparations are shown in A, B, C and $\mathrm{G}$. The level of sectioning is indicated by a black line in the specimens. (A-C) Neural vesicles induced after transplantation of the young head process of $\mathrm{HH} 5^{-}$ embryos. Note that their size and shape is comparable to the endogenous fore- and midbrains. Note the ectopic neural folds (arrowheads in B) and the thick neural epithelium. Note the restricted expression of $N K X 2.1$ in the ventral, CNOT1 in the dorsal and EN2 in the caudal domains. The rostral ends of the induced vesicles face the anterior pole of the primary embryos. (D,E) Neural vesicles induced by grafted prechordal plates (arrowheads) and head processes (arrows) from HH5 embryos. Note that only the vesicles generated by the prechordal plates express $N K X 2.1$ and CNOT1. (F) Neural vesicles induced by the prechordal plate (arrowhead) and the posterior head process (arrow) of $\mathrm{HH}^{+}$ embryos. Note ectopic EN2 expression is only seen in the vesicle induced by the posterior head process. (G) Neural vesicle induced by the HH6 prechordal plate. Note the small size, the thin neuroepithelium and the preponderance of the NKX2.1 expression. YHP, young head process; PP,

prechordal plate; HP, head process. Bar, $200 \mu \mathrm{m}$, except for the whole mount in $\mathrm{B}(300 \mu \mathrm{m})$. of EN2-positive cells (Fig. 1F, arrowhead). Transplantation of head processes of the same donor embryos (HH5, $n=5)$ resulted in neural vesicles, which were negative for NKX2.1 and CNOT1 (Fig. 1D,E, arrows). Posterior head processes of slightly older embryos (HH5 $\left.{ }^{+}, n=2\right)$ induced $E N 2$-expressing neural vesicles (Fig. 1F, arrow). Thus, the capacity to induce different anteroposterior levels of the brain segregates both temporally and spatially in parallel to the segregation of $G S C$ and $C N O T 1 / 2$ cells in the axial mesoderm. Prechordal plates from headfold-stage embryos (HH6, $n=17$ ) induced smaller neuroectodermal vesicles, indicating a decrease in neuralizing capacity (Fig. 1G, arrowhead). These vesicles contained between $50 \%$ and $90 \%$ of NKX2.1 positive cells, as compared to $5-50 \%$ resulting from younger prechordal plate grafts (HH5), indicating a relative increase of the ventralizing capacity.
NKX2.1
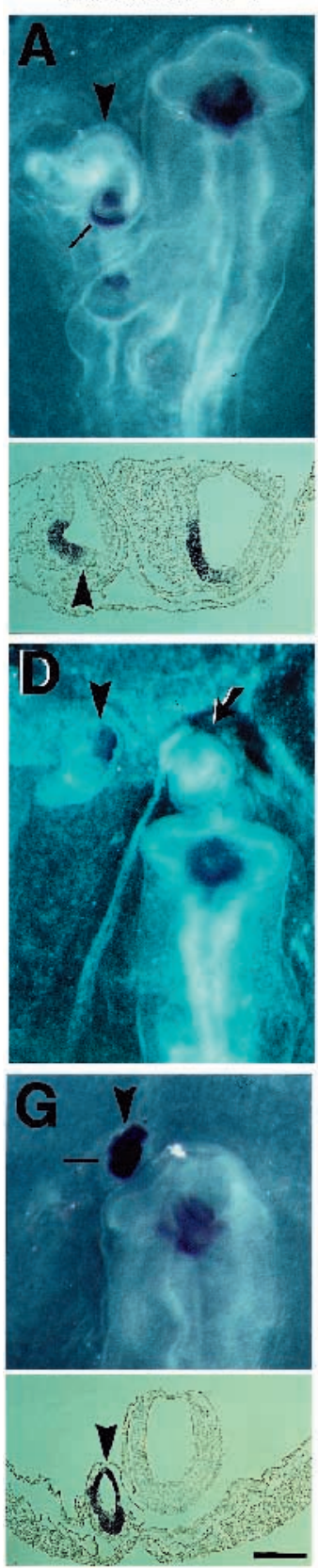

CNOT1
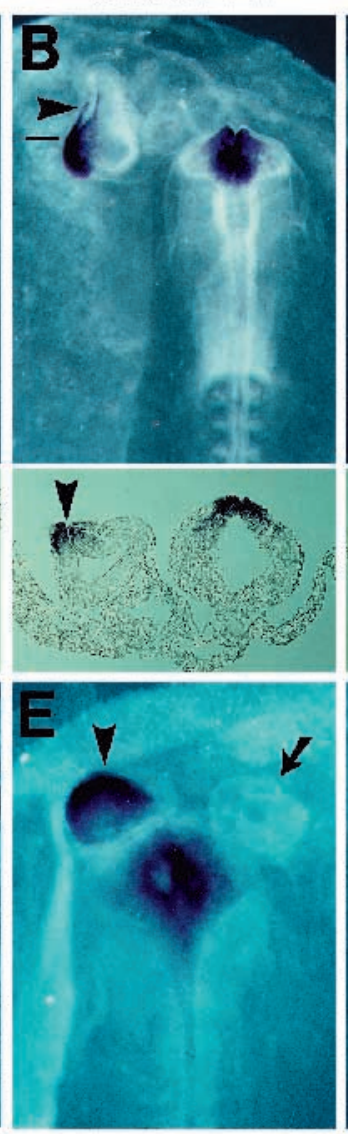

A, B,C

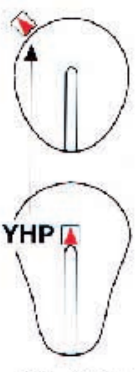

HH Q1.5 -
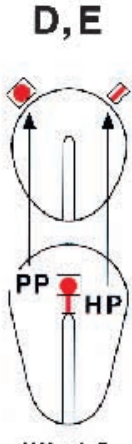

Hн $\$ 1.5$


$\mathbf{F}$

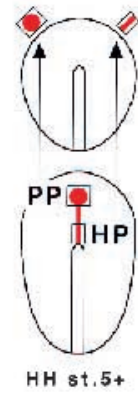

G

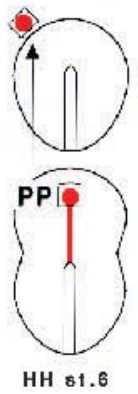


In summary, the young head process has the potential to induce fore- and midbrain, whereas the potential of the prechordal plate is restricted to the induction of forebrain.

\section{Ventralization of the developing forebrain by the prechordal plate}

We studied the effects of the anterior axial mesoderm on the lateral part of the anterior neural plate. The influence of ectopic young head processes or prechordal plates $\left(\mathrm{HH}^{-}-6\right)$ on forebrain development was analyzed after grafting laterally either anterior to the nodes (HH4, n=4, Fig. 3A) or slightly caudal to the endogenous prechordal plates (HH5-7; $n=17$, Fig. 2A). In these operations the host ectoderm was already neuralized at the sites of transplantation, as indicated by its differentiation capacity (Rao, 1968). We observed non-closure of the forebrains dorsally in host embryos at $\mathrm{HH}^{-}$to HH11 (Fig. 2B$\mathrm{E}$, arrowheads, 3B). The lateral diencephali including the optic evaginations were flattened on the operated sides (Figs 2B,D, $3 \mathrm{~B}$, arrows). This morphology suggested a loss of lateral structures and an overproduction of ventral structures, an interpretation corroborated by analysis of $N K X 2.1$ and $P A X 6$ expression. The forebrains showed ectopic $N K X 2.1$ and $S H H$ expression domains at the sides of the implants, either contiguous with the endogenous ventral domains or completely separated in the optic vesicles (Figs 2B,C, 3B,C). PAX6 expression was locally downregulated in the affected lateral regions of the forebrains (Fig. 2D,E).

We conclude that the prechordal plate is able to ventralize the forebrain not only in its natural, medial position, but also independently in the lateral neural plate.

\section{Influence of Sonic Hedgehog (SHH) on the developing forebrain in vivo}

SHH transcripts and proteins have been detected in the anterior axial mesoderm of chicken embryos (Johnson et al., 1994; Marti et al., 1995) and it is therefore an obvious candidate for a ventralizing factor in the prechordal region of the embryo. We confirmed transcription of $\mathrm{SHH}$ in the young head process $\left(\mathrm{HH}^{-}\right)$and the prechordal plate (HH5 and older). The neural ectoderm overlying the prechordal plate was negative up to HH8, when NKX2.1 expression appeared in the ventral forebrain anlage. At this stage, $S H H$ RNA was present caudal of the $N K X 2.1$ domain in the ventral part of the prospective midbrain. It appeared that the expression of $\mathrm{SHH}$ in the anterior neurectoderm depended on prior expression in the axial mesendoderm, similar to the situation in the epichordal area.

During $\mathrm{HH}$, $\mathrm{SHH}$ expression extended into the ventral forebrain, thereby overlapping with $N K X 2.1$ (HH10). Grafted young head processes and prechordal plates maintained $\mathrm{SHH}$ expression for at least 20 hours of incubation, regardless of the site of transplantation ( $n=5$, Fig. 3B,C, arrows). When the implant was grafted laterally in contact with the forebrain anlage, ectopic $S H H$ RNA could be detected in the adjacent region of the forebrain at $\mathrm{HH} 10(n=1$, Fig. $3 \mathrm{~A}-\mathrm{C})$. Thus $S H H$ is expressed in the prechordal plate endogenously and upon transplantation, during the time when the inducing activity of the prechordal plate is occurring.

In order to examine inductions mediated by $\mathrm{SHH}$ in vivo, we employed primary chicken fibroblasts infected with a $\mathrm{SHH}$ expressing, replication-competent retrovirus and non-infected primary chicken fibroblasts as negative controls. Due to the retroviral life cycle and the poor transcription in young embryos we did not expect nor observe viral propagation in our experiments. Retroviral spread would be reflected by ectopic SHH RNA in the host tissue, which we did not detect by in situ analysis ( $n=1$, Fig. 3D-F). Instead, at around 20 hours after implantation, virus-encoded $S H H$ transcripts were found exclusively in the grafted cells. This demonstrates that the virus did not propagate within the embryo. The infected cells were applied merely as strong producers of SHH protein. We labelled them with DiI for identification after transplantation and incubation. In order to control for SHH signalling, we cocultured infected and non-infected cells with rostral neural plate explants in collagen gels. In accordance with previously published data (Ericson et al., 1995), we found that under these conditions SHH-infected cells induced NKX2.1, while noninfected cells did not (not shown). We then checked the SHH

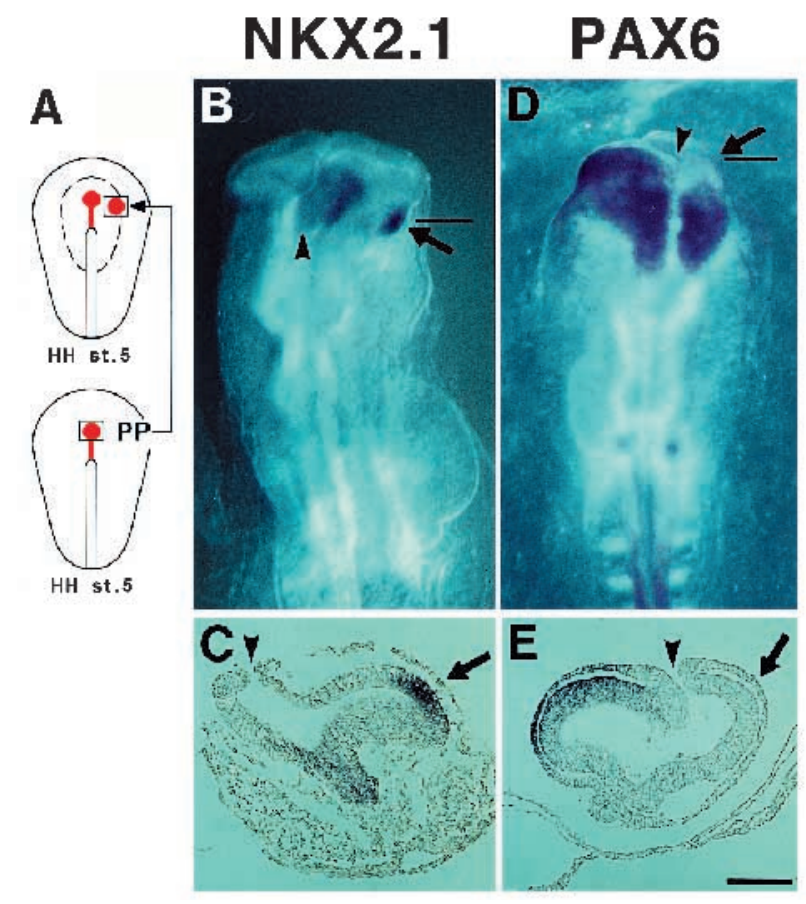

Fig. 2. Ventralization of the lateral forebrain by the prechordal plate. (A) Operation scheme. The graft of a HH5 donor embryo was laterally placed slightly posterior to the endogenous prechordal plate into an equally staged host. The dotted line in the host embryo demarcates the border of the prospective neural plate (Garcia-Martinez et al., 1993; Spratt, 1952). (B) Anterior part of the manipulated embryo at HH11 after whole-mount in situ analysis of NKX2.1 expression. Note that the forebrain failed to fuse dorsally (arrowhead). Compare the unilateral flattening of the forebrain on the operated side (right) with the indentation between the optic evagination and the posterior forebrain on the opposite side (left). Note the ectopic $N K X 2.1$ expression domain (arrow), which is clearly separate from the endogenous domain in the medial part of the forebrain. (C) Transverse section of the embryo in B at the level of the black line in B. Note the dorsal opening (arrowhead) and the ectopic $N K X 2.1$ expression domain (arrow) in the neural epithelium. (D) A similar embryo to that shown in B hybridized with a $P A X 6$ riboprobe as a marker for the lateral forebrain. Note the gap in the PAX6 expression domain (arrow) at the side of the implant.

(E) Transverse section of the embryo in $\mathrm{D}$ at the level of the black line in D. Note the unilateral lack of PAX6 expression (arrow) in the neuroepithelium and the overlying ectoderm. Bar, $200 \mu \mathrm{m}$ (B and D) and $120 \mu \mathrm{m}(\mathrm{C}$ and $\mathrm{E})$. 
cells for their neural-inducing capacity by transplantation into the rostral extraembryonic regions of host embryos. SHH cells did not induce any neuroepithelial structures or NKX2.1 expression ( $n=15$; not shown).

To examine whether $\mathrm{SHH}$ can ventralize the developing forebrain, we transplanted SHH cells laterally into host embryos (HH4- $5^{+}$) following the scheme of the experiments with the young head process and the prechordal plate $(n=46$, Figs $2 \mathrm{~A}$, $3 \mathrm{~A})$. The forebrain did not exhibit any morphological abnormalities or ectopic expression domains of $\mathrm{SHH}(n=1$, Fig. 3DF) and NKX2.1 ( $n=45$, not shown). During the incubation the majority of the grafted cells remained in close contact with the anterior neural plate up to $\mathrm{HH} 7$ only and were subsequently displaced to the ventral side. Therefore, we applied two other operation procedures in order to ensure sufficiently early and long contact to the forebrain anlage. We performed double implantations of $\mathrm{SHH}$ cells on cultured embryos, first at $\mathrm{HH} 4$ and for a second time at $\mathrm{HH} 5+(n=4)$. In addition, we transplanted SHH cells next to the lateral forebrain anlage in HH8 embryos by operating in ovo $(n=4)$. In no case did we observe ectopic $N K X 2.1$ expression. The only in vivo influences of $\mathrm{SHH}$ cells on forebrain development were observed after implantation close to the midline, rostral to the anterior axial mesoderm of HH5 embryos or under the headfold of HH6 embryos. This operation allowed the grafts to remain in contact with the developing forebrain until the time of analysis (HH10/11, n=5, Fig. $3 \mathrm{G}-\mathrm{I})$. In these cases a local distortion of the endogenous $N K X 2.1$ expression domain into the base of the optic evagination was induced, accompanied by localized morphological abnormalities. Ectopic NKX2.1 expression, however, was restricted to the proximal part of the forebrain. More distal regions remained $N K X 2.1$ negative, although in contact with the graft (Fig. 3I).

In summary, transplanted SHH cells could not induce an independent, lateral ventralization, as indicated by morphology and $S H H$ or $N K X 2.1$ expression domains. Only a distortion of the medial, endogeneous domain was inducible.

\section{Cyclopia after ablation of the prechordal plate}

The effect of the anterior axial mesendoderm or mesoderm on the development of the overlying brain was studied by extirpation experiments. We removed young head processes (HH5 ${ }^{-}$, $n=8)$, prechordal plates $\left(\mathrm{HH} 5 / 5^{+}, n=11\right)$, head processes alone $\left(\mathrm{HH} 5 / 5^{+}, n=9\right)$ or both prechordal plates plus head processes $\left(\mathrm{HH} 5 / 5^{+}, n=13\right)$, with the associated endoderm. The ectoderm was always left intact. After incubation to HH11, embryos were analyzed for expression of the ventral forebrain marker NKX2.1. The results of the specific operations were as follows:

\section{(1) Non-operated controls (Fig. 4A,F,I,K)}

Normal HH11 embryos display clearly separated optic evaginations at the lateral sides of the prosencephalon. These show PAX6 expression distally and are separated by a ventral, $N K X 2.1$ expression domain, which extends up to the caudal border of the prosencephalon (arrowhead). Dorsally the posterior border of the PAX6 expression domain coincides with the constriction between the forebrain and midbrain (arrowhead). The boundary between mesencephalon and metencephalon, the rhombencephalic isthmus, is clearly visible as an indentation (arrow) and is demarcated by the posterior border of $O T X 2$ expression.

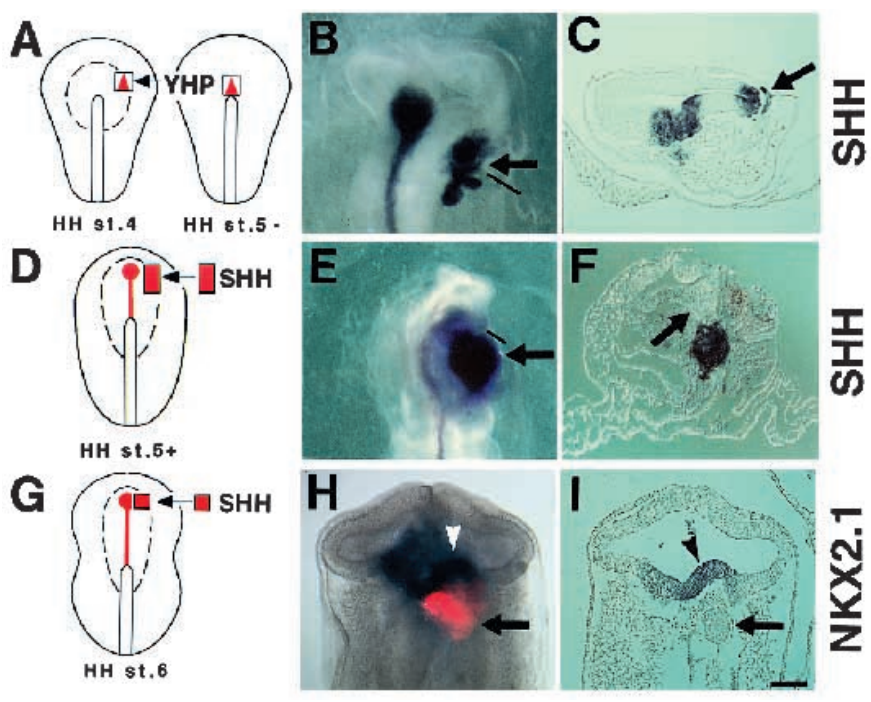

Fig. 3. Lateral ventralization by the young head process (YHP), but only medial ventralization by SHH-secreting cells. (A) Operation scheme showing the positioning of a YHP to the lateral neural plate. (B) Anterior region of the embryo (HH10) after whole-mount in situ analysis with a $\mathrm{SHH}$ riboprobe. Note the endogenous expression in the midline and the ectopic, neuroectodermal expression at the site of the implant (arrow). Black line indicates level of sectioning.

(C) Transverse section of embryo shown in B. The arrow points to SHH expression in the graft and induced expression in the adjacent neural epithelium. (D) Operation scheme demonstrating the grafting of SHH-secreting primary chick fibroblasts to the lateral neural plate. (E) Rostral part of the embryo (HH11) after whole-mount in situ hybridization with a $S H H$ riboprobe. Note the strong expression in the graft (arrow). Black line demarcates the level of sectioning. (F) Transverse section of the embryo in E. Note the lack of $\mathrm{SHH}$ RNA from the lateral forebrain close to the graft (arrow).

(G) Operation scheme showing the positioning of DiI-labelled SHH cells next to the prechordal plate of a host embryo. Note that the depicted operation scheme was the only condition that allowed ventralization of the forebrain by SHH cells in vivo. (H) Embryonic head after hybridization with a NKX2.1 riboprobe in a double exposure of epifluorescence and bright-field views. Note the local distortion of the $N K X 2.1$ expression domain (arrowhead) next to the red SHH cells (arrow). (I) Horizontal section of the embryo shown in $\mathrm{H}$. Overexpression of $N K X 2.1$ is restricted to the medial part of the forebrain (arrowhead). Note that the adjacent lateral part remains NKX2.1-negative, although it is in contact with the implant (arrow). Bar, $200 \mu \mathrm{m}$.

\section{(2) Extirpation of the young head process (Fig.} 4B,G,J,L).

This operation removed most of the prechordal plate cells. The eyefield did not become separated, resulting in a single optic evagination at the ventral side (cyclopia). The space inbetween the lateral PAX6 expression domains was severely reduced. Weak $N K X 2.1$ expression was induced more posteriorly than normal. The rostro-caudal extent of the forebrain and midbrain did not appear to be reduced and the mes-metencephalic isthmus still coincided with OTX2 expression.

\section{(3) Extirpation of the prechordal plate (Fig. 4C)}

In the absence of ventralized anterior prosencephalon (hypothalamus), the eyefield developed into a single optic evagination (cyclopia). The caudal prosencephalon exhibited strong 
NKX2.1 expression. The mesencephalon above the intact head process developed normally.

\section{(4) Extirpation of the head process (Fig. 4D,H)}

Since the prechordal plate remained untouched, the NKX2.1positive hypothalamus split the eyefield and no cyclopia formed. The caudal extension of the NKX2.1 expression domain was striking. PAX6 expression in the dorsal forebrain was not affected. The lateral constriction, a morphological landmark of the anterior neural tube, was shifted caudally, leaving a gap between it and the posterior border of the PAX6 domain.

\section{(5) Extirpation of prechordal plate plus head process (Fig. 4E)}

No expression of $N K X 2.1$ was induced, and the absence of ventral forebrain tissue resulted in a dramatically reduced dorso-ventral height of the diencephalon (not shown). Excluded from this reduction was the single optic evagination (cyclopia). The lateral constriction was shifted posteriorly.

It is obvious that removal of prechordal plate cells led to varying degrees of cyclopia (Fig. 4B,C,E,G,J). Here, the width of the

Fig. 4. Extirpation of the prechordal plate results in cyclopia, while the length of the neural tube is not reduced. (A-J) The rostral CNS of HH11 embryos after extirpation of different parts of the anterior axial mesendoderm or mesoderm (see operation schemes); ventral views (except for F and $\mathrm{H}$, which are shown as dorsal views). Embryos in A-E were hybridized with a $N K X 2.1$ probe, those in F-H with a PAX6 probe and those in I-J with an OTX2 probe, before the CNS was dissected free of surrounding tissues. Arrowheads indicate the lateral constriction in the anterior brain and arrows the mes-metencephalic isthmus. Note that cyclopia formation correlates with young head process (YHP) or prechordal plate (PP) removal. NKX2.1 expression correlates with the presence of the prechordal plate or the anterior head process (HP), which has not yet ingressed in $\mathrm{B}$ or is left intact in $\mathrm{C}$. Note the caudal extension of the $N K X 2.1$ expression, the posterior shift of the lateral constriction and the unaltered dorsal PAX6 expression upon ablation of the (young) head process. In G PAX6 expression in the posterior half of the forebrain is restricted to the dorsal region and clearly omits the ventral aspect. In no case is the rostrocaudal extent of the total length of foreand midbrain reduced upon extirpation, and the coincidence of posterior OTX2 expression and the mes-metencephalic isthmus is maintained. For further description and a discussion, see text. (K, L) Rostral neural tubes of HH11 embryos seen from ventral in scanning electron micrographs. Note the reduced forebrain width and the fusion of the optic evagiations ventrally upon removal of the young head process. Bar, $200 \mu \mathrm{m}$ (A-E) and 100 $\mu \mathrm{m}(\mathrm{F}$ and $\mathrm{G})$. forebrain was significantly reduced and the optic vesicles were barely or not at all separated. In contrast, neither cyclopia nor width reduction occurred when the prechordal plate remained in place and only the head process was removed. Remarkably, however, the rostro-caudal length of the forebrain-midbrain field, measured from the rhombencephalic isthmus to the

\section{CONTROL}
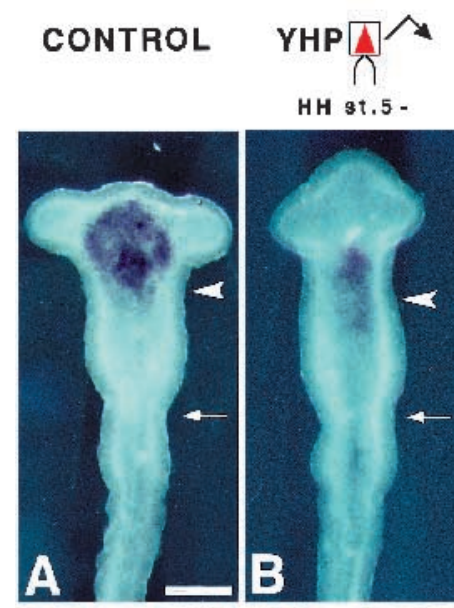

HH $3 t .5$ -
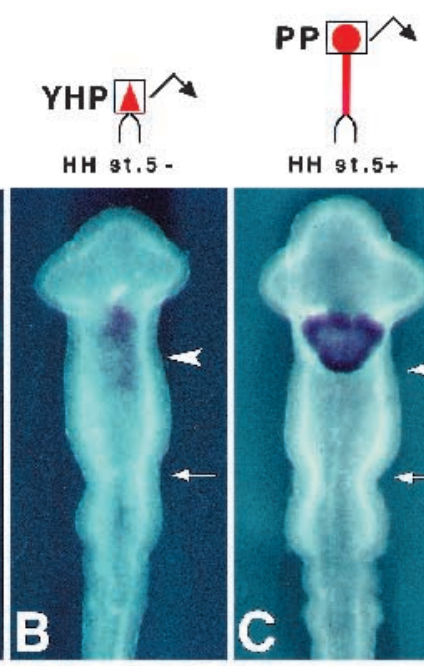

HH st.5+
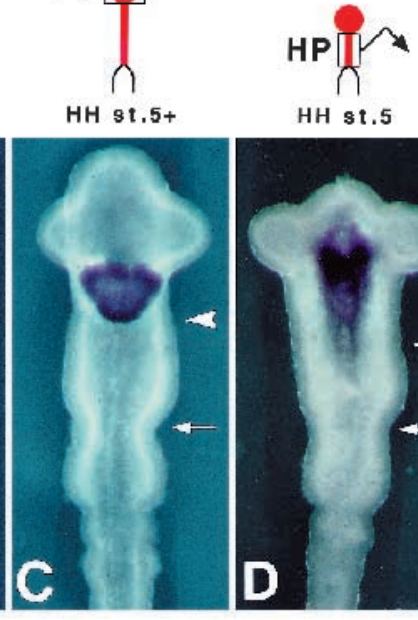

HH st.5
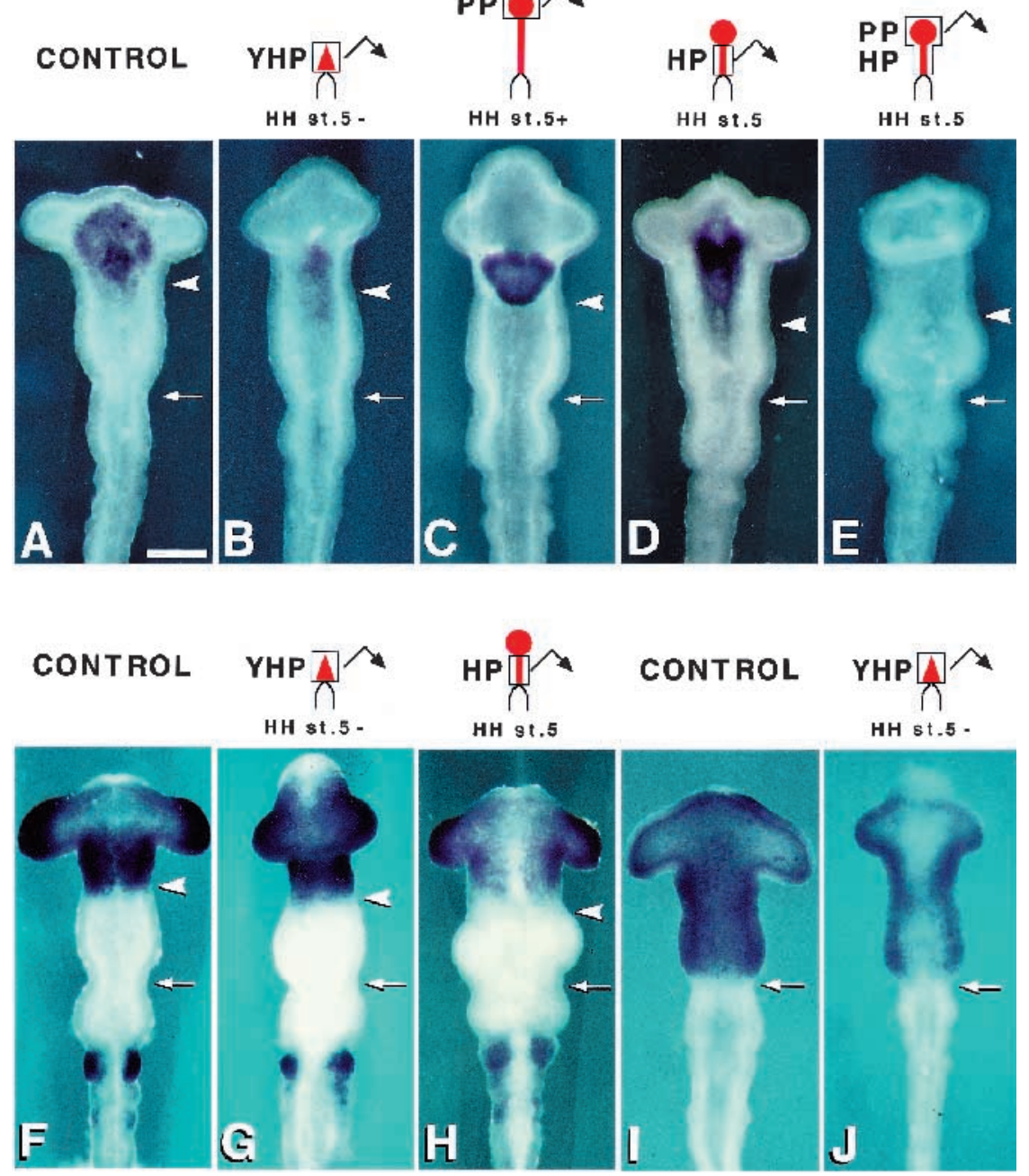


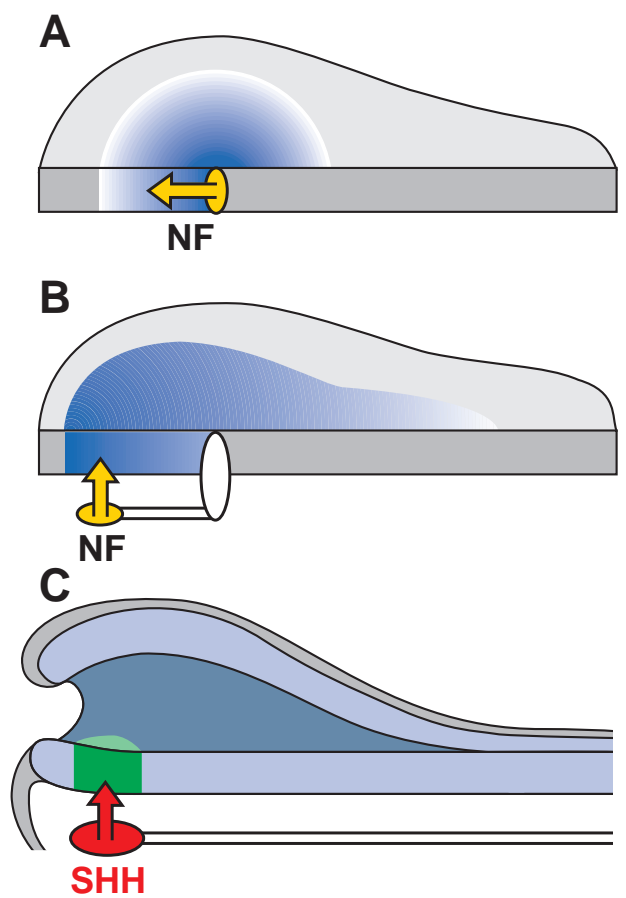

Fig. 5. Model for the patterning of the anterior neural ectoderm. The model outlines three distinct steps: (A) Anterior neural induction at the primitive streak stage. Neuralizing factors (NF, yellow) emanate from the node and spread in a planar fashion to antagonize BMP-4signals and induce the anterior neural ectoderm (blue). (B) Repatterning of the neural plate at the head process stage. The prechordal plate shifts the source of neuralizing factors (NF, yellow) anteriorly, where they act vertically and increase the relative concentration of BMP-4-antagonists. The medial part of the prospective forebrain is rendered susceptible for ventralizing factors. (C) Ventralization of the forebrain anlage at the 4-somite stage. The prechordal plate emits signals, including Sonic Hedgehog ( $\mathrm{SHH}$, red), which ventralize the forebrain anlage as shown by the induction of the ventral forebrain marker NKX2.1 (green).

anterior end of the neuraxis, was not reduced by any of these drastic manipulations.

In conclusion, extirpation of axial mesendoderm demonstrated a correlation between the correct location of a prechordal plate, the expression of NKX2.1, the generation of ventral prosencephalic tissue and the splitting of the eye anlagen. Furthermore, the experiments demonstrated that the rostro-caudal extension of the anterior neural tube does not depend on the axial mesendoderm or mesoderm.

\section{DISCUSSION}

\section{Neuralization}

The epiblast cells around Hensen's node are fated to become neural even before the primitive streak has reached its full elongation $\left(\mathrm{HH}^{+}\right)$. The young neural plate initially forms a circle around the tip of the primitive streak, and becomes pearshaped with further elongation of the embryo (HH4; GarciaMartinez et al., 1993; Spratt, 1952). The shape of the neural plate closely follows the shape of the growing endoderm (Vakaet, 1970), which could therefore represent a source for vertical signalling. According to Gallera, endoderm has no neural-inducing capacity (Gallera and Nicolet, 1969). An involvement of the primitive endoderm in head patterning, however, is indicated by the expression of the homeobox gene Hesxl in mice and the secreted factor cerberus in the frog Xenopus laevis (Bouwmeester et al., 1996; Thomas and Beddington, 1996). The location of the node in the center of the prospective neural plate suggests that it is the source of a neural-inducing signal spreading in a planar fashion. Candidate factors, which are expressed in organizer cells and known to act as direct neuralizing factors in Xenopus, are the BMPantagonists follistatin, noggin and chordin (HemmatiBrivanlou et al., 1994; Hemmati-Brivanlou and Melton, 1997; Lamb et al., 1993; Sasai et al., 1995). Neuralizing effects of these factors by BMP-antagonism have so far not been experimentally demonstrated in the chick; however, the expression of BMP4 in the chick epiblast, excluding the neural plate and anterior streak (Schultheiss et al., 1997; Watanabe and Le Douarin, 1996), strongly suggests a conserved neuralization mechanism based on BMP and antagonistic molecules.

We have demonstrated the capacity of the young head process $\left(\mathrm{HH}^{-}\right)$and the prechordal plate (HH5) to induce anterior neuroectoderm from naive epiblast in the area opaca. The application of molecular markers allowed early analysis long before regional differentiation took place. Our data are in agreement with the results obtained by K. Hara, who co-cultivated axial mesoderm with another kind of responding tissue, i.e. ectoderm from the area pellucida, and histologically analyzed the recombinates after 12 days of intracoelomic growth (Hara, 1961). Note, however, that early neural plate cells are already committed to neural development before formation of axial mesoderm (Rao, 1968). Our extirpation experiments have clearly shown that the rostro-caudal extension of the brain anlage is independent of the presence of the anterior axial mesoderm. The question remains: What is the biological significance of a second emission of neural-inducing factors by the prechordal plate, in addition to the organizerderived signalling?

\section{Competence for ventralization}

The forward migration of the prechordal plate cells translocates the source of neural inductive signalling from Hensen's node to a position around $400 \mu \mathrm{m}$ more anterior. Concomitantly, the distribution of neuralizing factors is expanded and the relative concentration of BMP antagonists versus BMP4 at the anterior margin of the neural plate is elevated. This changing pattern of the anterior neural plate in response to the prechordal plate is evident by the expression of the homeobox genes GSC, Lim 1 and $O t x 2$, which can be followed from the organizer to the anterior mesendoderm and to the overlying ectoderm (BallyCuif et al., 1995; Izpisúa-Belmonte et al., 1993; Shawlot and Behringer, 1995). We conclude that the change of pattern originating from the neuralizing activity of the prechordal plate is a prerequisite for a subsequent ventralization by SHH.

\section{Ventralization}

Compelling evidence suggests that SHH is the relevant ventralizing factor in the neural tube including the developing forebrain (Chiang et al., 1996). Our results show that the prechordal plate can ventralize the developing forebrain in a medial and lateral position, whereas infected fibroblasts 
expressing SHH can do so only close to the midline or in explanted neural plates. Similar findings have been described for more posterior regions of the neural tube with respect to the notochord, Sonic Hedgehog and floor plate induction. While a secondary notochord induces an ectopic floorplate in lateral regions of the chick neural tube (Yamada et al., 1991), overexpression of Sonic Hedgehog in frog embryos can expand the domain of ventral midline markers, but does not induce independent floorplate markers in lateral regions of alar or basal plates (Ruiz i Altaba et al., 1995). On the other hand, Sonic Hedgehog induces floorplate differentiation in rat lateral neural plate explants in vitro (Roelink et al., 1994). Thus, both in prechordal and epichordal regions of the embryo, a negative influence seems to be exerted in vivo from non-neural tissue, which could be the adjacent paraxial mesoderm or the lateral surface ectoderm. A candidate for the anti-neuralizing and antiSHH activity would be BMP4 (Liem et al., 1995; Schultheiss et al., 1997; Watanabe and Le Douarin, 1996). Three experimental conditions interfere with the inhibitory effect and raise the relative concentration of neural factors as a prerequisite for SHH action. One is to excise the neural plate, and thus mechanically remove vertical and lateral inhibitory influences (Ericson et al., 1995; Roelink et al., 1994). A second way is to achieve SHH signalling from within the neuroepithelium by expressing a Shh transgene in mice (Echelard et al., 1993) or frogs (Roelink et al., 1994). Our data suggest a third way, namely to 'medialize' the lateral neural plate by an ectopic prechordal plate, thus creating an ectopic source of neuralizing factors. In the light of this interpretation, the induction by the prechordal plate of a dorso-ventrally patterned neural vesicle (Fig. 1) and the lateral ventralization of the forebrain (Fig. 2) are two very related processes. The latter case could be regarded as an independent neural induction event within the neural plate, with subsequent formation of a common neural plate, but with two separate ventral domains. Both processes would require a neuralization step as a prerequisite for ventralization.

It is conceivable that additional factors are involved in the ventralization of the forebrain. BMP-7 is expressed in the rostral axial mesendoderm at the time the ventral forebrain is induced (Lyons et al., 1995; Arkell and Beddington, 1997). This distinguishes the young head process and the prechordal plate not only from the notochord, but also from SHHproducing cells. Further experiments will show whether BMP7 does indeed serve as the anteriorizing cofactor in ventral forebrain induction.

\section{The prechordal plate and cyclops formation}

According to fate-mapping studies the eyefield in vertebrates lies near the anterior margin of the neural plate (Couly and Le Douarin, 1985, 1987; Eagleson and Harris, 1990). In the chick the potential to differentiate eye structures is present at $\mathrm{HH} 4$, that is before the ingression of the prechordal plate (Khare and Choudury, 1985). Slightly later, at HH5, the eyefield becomes separated into two lateral parts (Rudnick, 1948). Since the prechordal plate invades the region below the eyefield, it appears to be crucial for its splitting. In the amphibian Amblystoma median rostral strips of the neural plate developed two bilateral eyes when co-transplanted with the archenteron roof (endomesoderm), but only one eye in the absence of the archenteron roof (Adelmann, 1929). Excision of the archenteron roof in the early neural plate stage of another amphibian, Triton, resulted in cyclopia (Mangold, 1931). In the present study we have dissected the function of the anterior axial mesendoderm spatially and temporally, and followed the ventral and dorsal forebrain identities with the expression of $N K X 2.1$ and PAX6 markers, respectively. We demonstrate the segregation of forebrain-inducing capacity with the prechordal plate tissue to a rostral position in the axial mesendoderm (Fig. 1). By removing the young head process (mostly prospective prechordal plate) or the prechordal plate itself, we demonstrate its role in splitting the eyefield of chicken embryos (Fig. 4). These results are in agreement with previous findings in frogs and chick embryos, which show that the prechordal plate interferes with retina formation by suppression of PAX6 expression ( $\mathrm{Li}$ et al., 1997). The involvement of SHH in this process is very probable since the Pax6 expression is restricted in zebrafish embryos, in which $S H H$ is overexpressed (Macdonald et al., 1995), and mice, which lack a functional SHH allele, are cyclopic (Chiang et al., 1996).

\section{The prechordal plate and the anlage of the hypothalamus and the hypophysis}

NKX2.1 demarcates the ventral forebrain, which later becomes the hypothalamus and the hypophysis (Lazzaro et al., 1991; Price et al., 1992). The ventral forebrain defects of homozygous mutant mice, namely the size reduction of the hypothalamus and the complete lack of hypophysis, show that not only is $N k x 2.1$ expressed in these structures, but it is essential for their formation (Kimura et al., 1996). The position of the endogenous $N K X 2.1$ expression domain dorsal of the prechordal plate suggests that vertical signals from the anterior axial mesoderm could be essential for the activity of $N K X 2.1$ in the ventral forebrain (Fig. 4). Insights into the mechanism underlying the patterning process, which eventually leads to the development of hypothalamus and hypophysis, are provided by transplantation experiments when the prechordal plate was grafted to the lateral side of the forebrain (Fig. 2). The ectopic expression of $N K X 2.1$ at the cost of PAX6 demonstrates that the prechordal plate affects the development of the anterior neural plate by transformation of a lateral default state into a ventral one. Thus, the role of the prechordal plate is not restricted to the splitting of the eyefield, but extends to the establishment of a new identity, namely the formation of hypothalamus and hypophysis.

\section{Evolution of the prechordal plate}

Two lower groups of chordates, ascidians (Urochordata) and Branchiostoma (Cephalochordata), each have a single eye, which is considered to be the homolog of the paired vertebrate eye (Lacalli, 1996; Lacalli et al., 1994; Salvini-Plawen and Mayr, 1977). They lack a prechordal plate, and their notochord is formed in continuity with the endoderm during gastrulation (Conklin, 1932; Satoh, 1994). We suggest that the existence of these naturally occurring cyclopic phenotypes is correlated with the absence of a prechordal plate.

Massive gene duplications must have occurred in evolution from the cephalochordates to the vertebrates (Holland et al., 1994). We have argued elsewhere that the generation of a prechordal plate in vertebrates might depend on the duplication of organizer-specific genes, in particular the homeobox gene goosecoid (Lemaire et al., 1997). In the chick we found a second goosecoid gene, 'GSX', which characterizes the early 
neural plate cells, while cells expressing the other GSC gene gastrulate and characterize the prechordal plate. We postulate that the patterning of the neural plate in the lower chordate Branchiostoma corresponds to the situation in a $\mathrm{HH} 4$ chick embryo, with the neural plate center coincident with the organizer and a single eyefield at its anterior margin. Further steps occurring later in phylogeny and ontogeny are the generation of a prechordal plate, the rostral translocation of the neural plate center and the splitting of the eyefield by elaboration of a ventral forebrain identity.

\section{A model for the role of the prechordal plate}

We propose the following model for the role of the prechordal plate in the patterning of the anterior neural ectoderm (Fig. 5). Three distinct steps are outlined, which culminate in the ventralization of the forebrain. First (Fig. 5A), anterior neuralization occurs at the primitive streak stage, when BMP-4-antagonizing factors emanate from the node and spread in a planar fashion to induce anterior neural ectoderm. Second (Fig. 5B), the anterior translocation of the organizer-derived cells shifts the source of neuralizing factors anteriorly, where the relative concentration of BMP-4-antagonists is thus elevated and the medial part of the prospective forebrain becomes competent to respond to ventralizing factors. Third (Fig. 5C), a Sonic Hedgehog signal, possibly in combination with BMP-7, ventralizes the forebrain anlage, reflected by the onset of $N K X 2.1$ expression at the 4-somite stage. A new identity, the prospective hypothalamus, is thereby created, splitting the eye anlage into two lateral domains.

We thank W. Behrens for excellent technical assistance, T. Marquardt for valuable support with the whole-mount in situ hybridization, R. Altschäffel for photography and H. Sebesse for graphic design. We thank T. Boettger, H. Böger, K. Ewan, P. Gruss, L. Lemaire, T. Roeser, T. Thomas, D. Treichel and A. Voss for discussions and comments on the manuscript and C. Gleed for linguistic improvements. The $S H H$ constructs were generously provided by C. Tabin, the OTX2 probe by E. Boncinelli. We gratefully acknowledge financial support by a DFG grant SFB271 and by the Max-Planck-Society.

\section{REFERENCES}

Adelmann, H. B. (1922). The significance of the prechordal plate. Am. J. Anat. 31, 55-101.

Adelmann, H. B. (1929). Experimental studies on the development of the eye. II. The eye-forming potencies of the median portions of the urodelan neural plate (Triton taeniatus and Amblystoma punctatum). J. exp. Zool. 54, 291317.

Arkell, R. and Beddington, R.S.P. (1997). BMP-7 influences pattern and growth of the developing hindbrain of mouse embryos. Development 124, 112

Bally-Cuif, L., Gulisano, M., Broccoli, V. and Boncinelli, E. (1995). c-otx2 is expressed in two different phases of gastrulation and is sensitive to retinoic acid treatment in chick embryos. Mech. Dev. 49, 49-63.

Barth, K. A. and Wilson, S. W. (1995). Expression of zebrafish nk2.2 is influenced by Sonic Hedgehog/vertebrate hedgehog-1 and demarcates a zone of neuronal differentiation in the embryonic forebrain. Development 121, 1755-1768.

Bouwmeester, T., Kim, S., Sasai, Y., Lu, B. and De Robertis, E. M. (1996). Cerberus is a head-inducing secreted factor expressed in the anterior endoderm of Spemann's organizer. Nature 382, 595-601.

Chiang, C., Litingtung, Y., Lee, E., Young, K. E., Corden, J. L., Westphal, H. and Beachy, P. A. (1996). Cyclopia and defective axial patterning in mice lacking Sonic Hedgehog gene function. Nature 383, 407-413.

Conklin, E. G. (1932). The embryology of Amphioxus. J. Morph. 54, 69-151.

Couly, G. F., Coltey, P. M. and Le Douarin, N. M. (1992). The developmental fate of the cephalic mesoderm in quail-chick chimeras. Development 114, 115.

Couly, G. F. and Le Douarin, N. M. (1985). Mapping of the early neural primordium in quail-chick chimeras. I. Developmental relationship between placodes, facial ectoderm and prosencephalon. Dev. Biol. 110, 422-439.

Couly, G. F. and Le Douarin, N. M. (1987). Mapping of the early neural primordium in quail-chick chimeras. II. The prosencephalic neural plate and neural folds: implications for the genesis of cephalic human congenital abnormalities. Dev. Biol. 120, 198-214.

De Robertis, E. M. (1995). Dismantling the organizer. Nature 374, 407-408.

Dias, M. S. and Schoenwolf, G. C. (1990). Formation of ectopic neuroepithelium in chick blastoderms: age related capacities for induction and self-differentiation following transplantation of quail Hensen's node. Anat. Rec. 229, 437-448.

Eagleson, G. W. and Harris, W. A. (1990). Mapping of the presumptive brain regions in the neural plate of Xenopus laevis. J. Neurobiol. 21, 427-440.

Echelard, Y., Epstein, D. J., St-Jacques, B., Shen, L., Mohler, J., McMahon, J. A. and McMahon, A. P. (1993). Sonic Hedgehog, a member of a family of putative signaling molecules, is implicated in the regulation of CNS polarity. Cell 75, 1417-1430.

Ericson, J., Muhr, J., Placzek, M., Lints, T., Jessell, T. M. and Edlund, T. (1995). Sonic Hedgehog induces the differentiation of ventral forebrain neurons: a common signal for ventral patterning within the neural tube. Cell 81, 747-756.

Fekete, D. M. and Cepko, C. L. (1993). Retroviral infection coupled with tissue transplantation limits gene transfer in the chicken embryo. Proc. Nat. Acad. Sci. USA 90, 2350-2354.

Gallera, J. and Nicolet, G. (1969). Le pouvoir inducteur de l'endoblaste presomptif contenu dans la ligne primitive jeune de poulet. J. Embryol. Exp. Morph. 21, 105-118.

Garcia-Martinez, V., Alvarez, I. S. and Schoenwolf, G. C. (1993). Locations of the ectodermal and nonectodermal subdivisions of the epiblast at stages 3 and 4 of avian gastrulation and neurulation. J. exp. Zool. 267, 431-446.

Gardner, C. A., Darnell, D. K., Poole, S. J., Ordahl, C. P. and Barald, K. F. (1988). Expression of an engrailed-like gene during development of the early embryonic chick nervous system. J. Neurosci. Res. 21, 426-437.

Hamburger, V. and Hamilton, H. L. (1951). A series of normal stages in the development of the chick embryo. J. Morph. 88, 49-92.

Hara, K. (1961). Regional neural differentiation induced by prechordal and presumptive chordal mesoderm in the chick embryo. $\mathrm{PhD}$ dissertation, University of Utrecht.

Hauptmann, G. and Gerster, T. (1996). Complex expression of the zp-50 pou gene in the embryonic zebrafish brain is altered by overexpression of Sonic Hedgehog. Development 122, 1769-1780.

Hayat, M. A. (1978). Introduction to Biological Scanning Electron Microscopy. University Park Press: Baltimore, London, Tokyo.

Hemmati-Brivanlou, A., Kelly, O. G. and Melton, D. A. (1994). Follistatin, an antagonist of activin, is expressed in the Spemann organizer and displays direct neuralizing activity. Cell 77, 283-295.

Hemmati-Brivanlou, A. and Melton, D. (1997). Vertebrate embryonic cells will become nerve cells unless told otherwise. Cell 88, 13-17.

Holland, P. W., Garcia-Fernandez, J., Williams, N. A. and Sidow, A. (1994). Gene duplications and the origins of vertebrate development. Development Supplement 125-133.

Izpisúa-Belmonte, J. C., De Robertis, E. M., Storey, K. G. and Stern, C. D. (1993). The homeobox gene goosecoid and the origin of organizer cells in the early chick blastoderm. Cell 74, 645-659.

Johnson, R. L., Laufer, E., Riddle, R. D. and Tabin, C. (1994). Ectopic expression of Sonic Hedgehog alters dorsal-ventral patterning of somites. Cell 79, 1165-1173.

Khare, M. K. and Choudury, S. (1985). Establishment of the neural differentiation pattern in the prospective prosencephalic ectoderm of the chick embryo (Gallus domesticus). Dev. Growth Differ. 27, 83-93.

Kimura, S., Hara, Y., Pineau, T., Fernandez-Salguero, P., Fox, C. H., Ward, J. M. and Gonzalez, F. J. (1996). The T/ebp null mouse: thyroid-specific enhancer-binding protein is essential for the organogenesis of the thyroid, lung, ventral forebrain and pituitary. Genes Dev. 10, 60-69.

Krauss, S., Concordet, J. P. and Ingham, P. W. (1993). A functionally conserved homolog of the Drosophila segment polarity gene hh is expressed in tissues with polarizing activity in zebrafish embryos. Cell 75, 1431-1444.

Lacalli, T. C. (1996). Frontal eye circuitry, rostral sensory pathways and brain organization in amphioxus larvae: evidence from 3D reconstructions. Phil. Trans. R. Soc. Lond. B 351, 243-263.

Lacalli, T. C., Holland, N. D. and West, J. E. (1994). Landmarks in the 
anterior central nervous system of amphioxus larvae. Phil. Trans. R. Soc. Lond. B 344, 165-185.

Lamb, T. M., Knecht, A. K., Smith, W. C., Stachel, S. E., Economides, A. N., Stahl, N., Yancopolous, G. D. and Harland, R. M. (1993). Neural induction by the secreted polypeptide noggin. Science 262, 713-8.

Lazzaro, D., Price, M., De Felice, M. and Di Lauro, R. (1991). The transcription factor TTF-1 is expressed at the onset of thyroid and lung morphogenesis and in restricted regions of the foetal brain. Development 113, 1093-1104.

Lemaire, L., Roeser, T., Izpisua-Belmonte, J.-C. and M. K. (1997). Segregating expression domains of two goosecoid genes during the transition from gastrulation to neurulation in chick embryos. Development 124, 14431452.

Li, H., Tierney, C., Wen, L., Wu, J. Y. and Rao, Y. (1997). A single morphogenetic field gives rise to two retina primordia under the influence of the prechordal plate. Development 124, 603-615.

Li, H.-S., Yang, J.-M., Jacobson, R. D., Pasko, D. and Sundin, O. (1994). Pax-6 is first expressed in a region of ectoderm anterior to the early neural plate: implications for stepwise determination of the lens. Dev. Biol. 162, 181-194.

Liem, K. F., Jr., Tremml, G., Roelink, H. and Jessell, T. M. (1995). Dorsal differentiation of neural plate cells induced by BMP-mediated signals from epidermal ectoderm. Cell 82, 969-979.

Lyons, K. M., Hogan, B. M. L. and Robertson, E. J. (1995). Colocalization of BMP-7 and BMP-2 RNAs suggests that these factors cooperatively mediate tissue interactions during murine development. Mech. Dev. 50, 71-83.

Macdonald, R., Barth, K. A., Xu, Q., Holder, N., Mikkola, I. and Wilson, S. W. (1995). Midline signalling is required for Pax gene regulation and patterning of the eyes. Development 121, 3267-3278.

Mangold, O. (1931). Das Determinierungsproblem. III. Das Wirbeltierauge in der Entwicklung und Regeneration. Ergebn. Biol. 7, 193-403.

Marti, E., Takada, R., Bumcrot, D. A., Sasaki, H. and McMahon, A. P. (1995). Distribution of Sonic Hedgehog peptides in the developing chick and mouse embryo. Development 121, 2537-2547.

Meier, S. (1981). Development of the chick embryo mesoblast: morphogenesis of the prechordal plate and cranial segments. Dev. Biol. 83, 49-61.

Niehrs, C., Keller, R., Cho, K. W. Y. and De Robertis, E. M. (1993). The homeobox gene goosecoid controls cell migration in Xenopus embryos. Cell 72, 491-503.

Piccolo, S., Sasai, Y., Lu, B. and De Robertis, E. M. (1996). Dorsoventral patterning in Xenopus: Inhibition of ventral signals by direct binding of chordin to BMP-4. Cell 86, 589-598.

Placzek, M., Jessell, T. M. and Dodd, J. (1993). Induction of floor plate differentiation by contact-dependent, homeogenetic signals. Development 117, 205-218.

Placzek, M., Tessier-Lavigne, M., Yamada, T., Jessel, T. and Dodd, D. (1990). Mesodermal control of neural cell identity: Floor plate induction by the notochord. Science 250, 985-988.

Potts, W. M., Olsen, M., Boettiger, D. and Vogt, V. M. (1987). Epitope mapping of monoclonal antibodies to gag protein p19 of avian sarcoma and leukaemia viruses. J. Gen. Virol. 68, 3177-3182.

Price, M., Lazzaro, D., Pohl, T., Mattei, M.-G., Rüther, U., Olivo, J.-C., Duboule, D. and Di Lauro, R. (1992). Regional expression of the homeobox gene $N k x-2.2$ in the developing mam malian forebrain. Neuron 8, 241-255.

Rao, B. R. (1968). The appearance and extension of neural differentiation tendencies in the neuroectoderm of the early chick embryo. Wilhelm Roux' Archiv. 160, 187-236.

Riddle, R. D., Johnson, R. L., Laufer, E. and Tabin, C. (1993). Sonic Hedgehog mediates the polarizing activity of the ZPA. Cell 75, 1401-1416.

Roelink, H., Augsburger, A., Heemskerk, J., Korzh, V., Norlin, S., Ruiz i Altaba, A., Tanabe, Y., Placzek, M., Edlund, T.and Jessell, T. M. (1994). Floor plate and motor neuron induction by vhh-1, a vertebrate homolog of hedgehog expressed by the notochord. Cell 76, 761-775.

Rudnick, D. (1948). Prospective areas and differentiation potencies in the chick blastoderm. Ann. NY Acad. Sci. 49, 761-772.

Ruiz i Altaba, A. (1993). Induction and axial patterning of the neural plate: planar and vertical signals. J. Neurobiol 24, 1276-1304.

Ruiz i Altaba, A., Jessell, T. M. and Roelink, H. (1995). Restrictions to floor plate induction by hedgehog and winged-helix genes in the neural tube of frog embryos. Mol. Cell. Neurosci. 6, 106-121.

Salvini-Plawen, L. V. and Mayr, E. (1977). On the evolution of photoreceptors and eyes. In Evolutionary Biology 10 (ed. Dobzhansky, T. et al.), pp. 207263. Plenum Press: New York.
Sasai, Y., Lu, B., Steinbeisser, H. and De Robertis, E. M. (1995). Regulation of neural induction by the Chd and Bmp-4 antagonistic patterning signals in Xenopus. Nature 376, 333-336.

Satoh, N. (1994). Developmental Biology of Ascidians. Cambridge University Press: Cambridge, UK.

Schoenwolf, G. C. and Smith, J. L. (1990). Mechanisms of neurulation: traditional viewpoint and recent advances. Development 109, 243-270.

Schultheiss, T. M., Burch, J. B. and Lassar, A. B. (1997). A role for bone morphogenetic proteins in the induction of cardiac myogenesis. Genes Dev. 11, 451-462.

Seifert, R., Jacob, M. and Jacob, H. J. (1993). The avian prechordal head region: a morphological study. J. Anat. 183, 75-89.

Shawlot, W. and Behringer, R. R. (1995). Requirement for Liml in headorganizer function. Nature 374, 425-430.

Shimamura, K., Hartigan, D. J., Martinez, S., Puelles, L. and Rubenstein, J. L. (1995). Longitudinal organization of the anterior neural plate and neural tube. Development 121, 3923-3933.

Slack, J. M. W. and Tannahill, D. (1992). Mechanisms of anteroposterior axis specification in vertebrates. Development 114, 285-302.

Smith, J. L. and Schoenwolf, G. C. (1989). Notochoral induction of cell wedging in the chick neural plate and its role in neural tube formation. J. Exp. Zool. 250, 49-62.

Spemann, H. and Mangold, H. (1924). Über die Induktion von Embryoanlagen durch Implantation artfremder Organisatoren. Roux' Arch. Entwiclungsmech. 100, 599-638.

Spratt, N. T. (1952). Localization of the prospective neural plate in the early chick blastoderm. J. Exp. Zool. 120, 109-130.

Stein, S. and Kessel, M. (1995). A homeobox gene involved in node, notochord and neural plate formation of chick embryos. Mech. Dev. 49, 37-48.

Stein, S., Niß, K. and Kessel, M. (1996). Differential activation of the clustered homeobox genes CNOT2 and CNOT1 during notogenesis in the chick. Dev. Biol. 180, 519-533.

Stern, C. D. (1993). Avian Embryos, pp. 45-54. IRL Press: Oxford.

Storey, K. G., Crossley, J. M., De Robertis, E. M., Norris, W. E. and Stern, C. D. (1992). Neural induction and regionalisation in the chick embryo. Development 114, 729-741.

Streit, A., Sockanathan, S., Perez, L., Rex, M., Scotting, P. J., Sharpe, P. T., Lovell-Badge, R. and Stern, C. D. (1997). Preventing the loss of competence for neural induction: HGF/SF, L5 and Sox-2. Development 124, 1191-1202.

Sulik, K., Dehart, D. B., Iangaki, T., Carson, J. L., Vrablic, T., Gesteland, K. and Schoenwolf, G. C. (1994). Morphogenesis of the murine node and notochordal plate. Dev. Dynam. 201, 260-278.

Thomas, P. and Beddington, R. (1996). Anterior primitive endoderm may be responsible for patterning the anterior neural plate in the mouse embryo. Curr. Biol. 6, 1487-1496.

Vakaet, L. (1970). Cinephotomicrographic investigations of gastrulation in the chick blastoderm. Arch. Biol. 81, 387-426.

van Straaten, H. W., Hekking, J. W., Wiertz-Hoessels, E. J., Thors, F. and Drukker, J. (1988). Effect of the notochord on the differentiation of a floor plate area in the neural tube of the chick embryo. Anat. Embryol. 177, 317324.

Wachtler, F., Jacob, H. J., Jacob, M. and Christ, B. (1984). The extrinsic ocular muscles in birds are derived from the prechordal plate. Naturwissenschaften 71, 379-380.

Watanabe, Y. and Le Douarin, N. M. (1996). A role for BMP-4 in the development of subcutaneous cartilage. Mech. Dev. 57, 69-78.

Wilkinson, D. G. (1992). In Situ Hybridisation; A Practical Approach. Oxford University Press: London.

Yamada, T., Placzek, M., Tanaka, H., Dodd, J. and Jessell, T. M. (1991). Control of cell pattern in the developing nervous system: Polarizing activity of the floor plate and notochord. Cell 64, 635-647.

Yamashita, H., ten Dijke, P., Huylebroeck, D., Sampath, T. K., Andries, M., Smith, J. C., Heldin, C. H. and Miyazono, K. (1995). Osteogenic protein-1 binds to activin type II receptors and induces certain activin-like effects. $J$. Cell Biol. 130, 217-26.

Zimmermann, L. B., De Jesus-Escobar, J. M. and Harland, R. M. (1996). The Spemann organizer signal noggin binds and inactivates bone morphogenetic protein 4. Cell 86, 599-606. 